\title{
Studies on Anionic Surfactant Structure in the Aggregation with (Hydroxypropyl)cellulose
}

\author{
Ricardo M. de Martins, Carolina A. Silva, Cristiane Becker, Dimitrios Samios, Clara I. D. Bica, Marcelo Christoff \\ Instituto de Química, UFRGS
}

\begin{abstract}
Fluorescence probing, viscosity and light scattering measurements have been combined to study the aggregation of different anionic surfactants mainly in dilute solutions $(0.5 \% \mathrm{w} / \mathrm{v})$ of (hydroxypropyl)cellulose (HPC MW 173,000), in moderate ionic strength $(\mathrm{NaCl} 0.1$ mol.L-1 $)$. The set of surfactants includes natural cholesterol derivatives, sodium cholate (CS) and sodium deoxycholate (DC), and the alkylsulphate, sodium dodecylsulphate (SDS). At $298 \mathrm{~K}$ the critical surfactant concentration related to aggregate/HPC formation $\left(\mathrm{C}_{1}\right)$ decreases for SDS and DC whereas it increases slightly for CS. At $312 \mathrm{~K}$ the $\mathrm{C}_{1}$ values for CS and DC are slightly shifted toward higher values whereas it is not changed for SDS. All surfactant/HPC systems increase $\mathrm{C}_{1}$ values as the HPC concentration increases to $1.2 \%$. Above $\mathrm{C}_{1}$ the viscosity increases for all surfactant/HPC systems but it is sharper in the increasing order CS, DC and SDS. The hydrodynamic behavior indicates that CS induces higher diffusion to HPC than SDS and DC. The aggregation in the surfactant/HPC systems is analyzed through the feature of surfactant/aggregate structure (size, charge density, etc).
\end{abstract}

Keywords: Aggregate, bile salt, hydrophobic, (hydroxypropyl)cellulose, fluorescence, light scattering, viscosity

\section{Introduction}

Cellulose ether derivatives as (hydroxypropyl) cellulose (HPC) are water-soluble uncharged polymers that interact with anionic surfactants in solution resulting in special rheological properties to the system. The understanding of the mechanisms operating and structures formed in such solutions as well as the numerous applications of non-ionic polymer-ionic surfactant systems in the pharmaceutical formulations, cosmetics, enhanced oil recovery, paint and food products have contributed to several studies on these systems over the last 30 years. Interactions between nonionic polymers and ionic surfactants are a consequence of weak intermolecular forces, which allow for a wide variety and range of behaviors in these systems. The directed action of a number of weak interactions leads to the cooperative nature of the aggregation behavior usually characterized by a starting surfactant concentration termed the critical aggregation concentration $\left(\mathrm{C}_{1}\right)$. The stabilization of the interfaces between the hydrophobic core of the aggregates and water is the major driving force for polymer-surfactant interaction $^{[1,2]}$.

Different research groups have focused on the relations between structure, charge and hydrophobicity of surfactants and polymers. Many studies have explored hydrophobic modifications in the polymers, but only a few types of surfactants have been investigated, most of them formed by long alkyl chains with polar head group ${ }^{[3-6]}$. We have recently studied different anionic surfactants with respect to the aggregation with low molar mass, non charged and flexible polymers as poly(ethylene oxide) and poly(vinyl pyrrolidone) ${ }^{[7,8]}$. These studies included some natural anionic surfactant bile salts, sodium cholate (CS) and sodium deoxycholate (DC), that are carboxylic polyhydroxy derivatives from cholestero [ ${ }^{[9]}$ and the well-known sodium dodecylsulphate (SDS) that is a long chain alkylsulphate. The molecular shape of the bile salts exhibits a planar polarity due to spatial distribution of lateral groups in their steroid backbone. The bile salt structures result in smaller and more rigid aggregates than the micelles formed by conventional alkylsurfactants, providing highly non

Autor para correspondência: Marcelo Christoff, Departamento de Físico-Química, UFRGS/IQ, Caixa Postal 15003, CEP: 91501-970, Porto Alegre, RS. E-mail:mchristo@portoweb.com.br 
polar microenvironments with chiral properties ${ }^{[10]}$. Indeed, the balance of hydrophobic and electrostatic forces in the polymer-surfactant interaction in moderate ionic strength was shown sensitive to different surfactant structures being the hydrophobic component more relevant to bile salt aggregation ${ }^{[7,8]}$.

The present work extends the studies with the same set of surfactants (CS, DC and SDS) (Scheme 1) to compare their aggregation behavior in the presence of the more rigid non-ionic polymer HPC at different temperatures. The HPC/SDS system has been shown as possessing the highest surfactant affinity to the cellulose ether derivative by fluorescence probing light scattering and capilar viscometry techniques ${ }^{[7,8]}$.

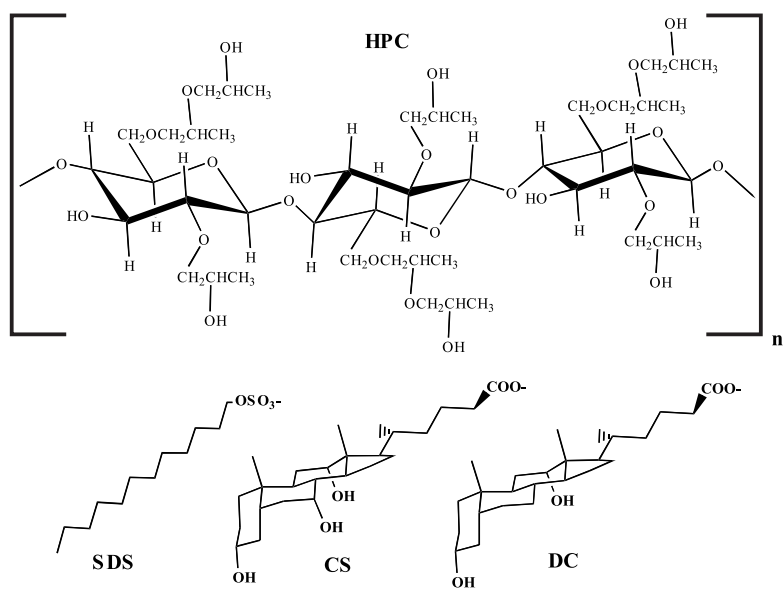

Scheme 1

\section{Experimental}

The surfactants DC(Sigma), CS(Sigma), SDS (Fischer) were used as received. The HPC (Aldrich) presents $\overline{\mathrm{M}}_{\mathrm{w}} \cong 173,000 ; \overline{\mathrm{M}}_{\mathrm{w}} / \overline{\mathrm{M}}_{\mathrm{n}}=3.5$ (GPC); and the average molar substitution for hydroxypropyl oxide group per anhydroglucose unit, $\mathrm{MS}=4.5$ (NMR). A stock aqueous solution of HPC was dialysed one week (Membracel tubing, cut-off 12000-16000; Polylabo) and filtered subsequently through 8 and $0.45 \mu \mathrm{m}$ membrane filters (Millipore). Pyrene (Py, Aldrich) was recrystallized twice from ethanol solutions. All solutions were prepared with Milli-Q grade water (Millipore). The probe solution was prepared by evaporating the suitable volume of the ethanol stock solution, followed by dissolution of the remaining solid in the surfactant/HPC solution. All surfactant/ HPC solutions were stirred for $12 \mathrm{~h}$ at room temperature before the measurements. To remove dust for the light scattering experiments the solutions were filtered through $0.45 \mu \mathrm{m}$ filter (Millipore) and centrifuged at $4000 \mathrm{rpm}$ for $90 \mathrm{~min}$. All the presented data are averaged from 3 experimental sets.

Fluorescence: The steady-state fluorescence measurements employed a Hitachi F-4500 Spectrophotometer with cell holder thermostated by a circulating ethylene glycol bath. The fluorescence spectra for Py were recorded in the corrected spectrum mode with excitation wavelength set at $336 \mathrm{~nm}$ and 2.5 $\mathrm{mm}$ slit. The ratio $\mathrm{I}_{1} / \mathrm{I}_{3}$ was taken from first $(372 \mathrm{~nm})$ and third $(384 \mathrm{~nm})$ vibronic peaks in the Py emission spectrum that has been shown to be a sensitive function of local polarity in organized systems ${ }^{[11]}$ (Figure 1). The Py concentration was kept $\leq 5 \times 10^{-6}$ mol. $\mathrm{L}^{-1}$ to exclude the possibility of excimer formation $\left(10^{-4} \mathrm{M}\right)$.

Light Scattering: Static light scattering and photon correlation measurements have been undertaken on a Brookhaven Instruments spectrometer, with a He-Ne laser at $632.8 \mathrm{~nm}$. Intensities were correlated by a 264-channel BI-9000 AT correlator covering 5 decades in delay time. The samples were thermostated in a refractive-index-matching liquid (decaline). In order to characterize the hydrodynamic behavior of the aggregates the multisampling time autocorrelation functions were analyzed by inverse Laplace transformation using the CONTIN ${ }^{[12]}$ and by two-exponential fits (Microcal Origin 6.0). All experiments took place at $298 \mathrm{~K}$.

Viscosity: The viscometric measurements were carried out in ordinary Cannon Fenske capillary viscometer $\left(\mathrm{NaCl} 0.1\right.$ mol.L $\mathrm{L}^{-1}$ flow time $\sim 79 \mathrm{~s}$ ) thermostated at $298 \mathrm{~K}$ by water bath.

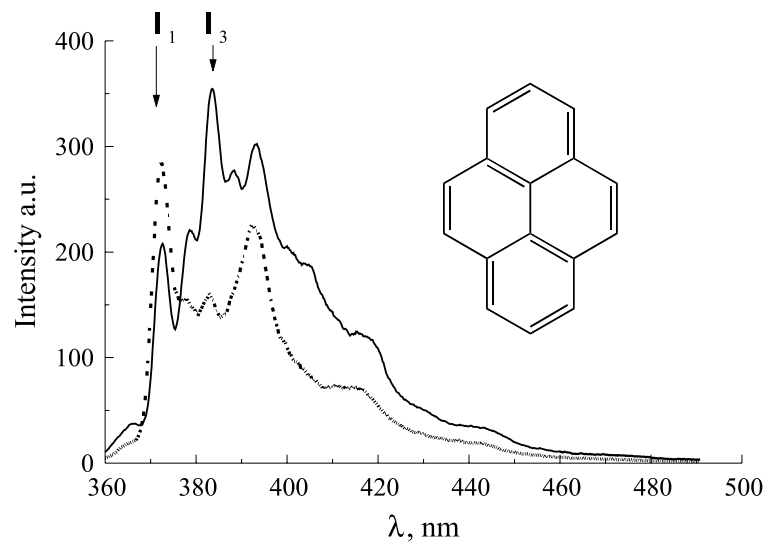

Figure 1. Fluorescence spectra of Pyrene $\left(10^{-6} \mathrm{~mol} . \mathrm{L}^{-1}\right)$ in cyclohexane $\left(\mathrm{I}_{1} / \mathrm{I}_{3} \approx 0.6\right.$, solid line $)$, and in water $\left(\mathrm{I}_{1} / \mathrm{I}_{3} \approx 1.9\right.$, dash line $)$. Insert: molecular structure of pyrene . 
The relative viscosity, $\eta_{\text {rel }}$ was obtained by the expression $\eta_{\text {rel }}=\eta / \eta_{0}$ where $\eta$ and $\eta_{0}$ are the viscosities of the sample and $0.5 \% \mathrm{HPC} / \mathrm{NaCl} 0.1$ mol.L $L^{-1}$ solution, respectively. Densities were determined using hydrometers from Arthur $\mathrm{H}$. Thomas Co. The value of the intrinsic viscosity, [ $\eta]$ was obtained by extrapolation of the specific viscosity, $\eta_{\mathrm{sp}}=\eta_{\mathrm{re}} \mathrm{e}^{-1} / \mathrm{c}$ to zero polymer concentration, $\mathrm{c}=0$. The overlap concentration, $\mathrm{c}^{*}$ for $\mathrm{HPC} / \mathrm{NaCl} 0.1$ mol. $L^{-1}$ was determined as $1 /[\eta] \cong 0.8 \%$ (not shown).

\section{Results and Discussion}

Fluorescence: The ratio $\mathrm{I}_{1} / \mathrm{I}_{3}$ from Py fluorescence decreases for different systems as the total surfactant concentration increases reflecting the incorporation of Py in a hydrophobic site as the aggregate is formed (Figure 2). The critical surfactant concentration $\left(\mathrm{C}_{1}\right)$ was determined by the intercept from linear extrapolation on bottom and steep data $\left(\mathrm{C}_{1}{ }^{\mathrm{A}}\right)$ or by the inflection point of the curve $\left(\mathrm{C}_{1}{ }^{\mathrm{B}}\right)$ (Table 1). The critical surfactant concentration without polymer (critical micellization concentration, $\mathrm{cmc}$ ) was obtained for each surfactant at $298 \mathrm{~K}$ in good agreement with literature values as $2.0 \mathrm{~mol}^{-\mathrm{L}^{-1}}$ for

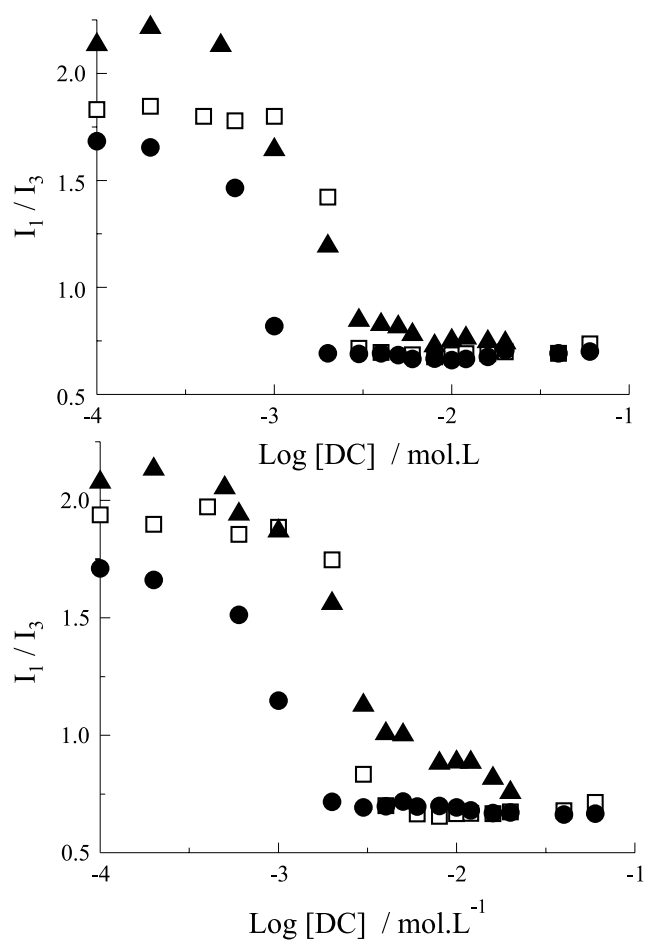

Figure 2. Dependence of the ratio $I_{1} / I_{3}$ on total DC concentration in $\mathrm{NaCl} 0.1$ mol.L $\mathrm{L}^{-1}$ aqueous solution, in absence ( $\square$ ) or with HPC $0.5 \%$ $(\bullet)$ and $1.2 \%(\mathbf{\Lambda})$; on top at $298 \mathrm{~K}$, bottom at $312 \mathrm{~K}$.
$\mathrm{SDS}^{[13]}, 3.1 \mathrm{~mol} . \mathrm{L}^{-1}$ for DC ${ }^{[6]}$, and $9.3 \mathrm{~mol} . \mathrm{L}^{-1}$ for $\mathrm{CS}^{[6]}$. In the presence of $0.5 \% \mathrm{HPC}$ both $\mathrm{C}_{1}$ values decrease with respect to $\mathrm{cmc}$ either for $\mathrm{DC}$ or $\mathrm{SDS}^{[14,15]}$ whereas a small increase is observed for CS. The former effect does point to polymer and surfactant association but the latter does not exclude the interaction $^{[16]}$. An increase of the critical surfactant aggregation reflects the destabilization of the micelle, which could be attributed to dispersion of the surfactant monomers along the polymer chains. Indeed, increasing the HPC concentration from $0.5 \%$ to $1.2 \% \mathrm{w} / \mathrm{v}$ (semi-dilute regime) all surfactants increase the $\mathrm{C}_{1}$ with respect to lower HPC solution. The same surfactants set has been recently showed a similar behavior with poly(ethylene oxide) $)^{[7,8]}$.

The micropolarity $(\mu \mathrm{P})$, as the average ratio $\mathrm{I}_{1} / \mathrm{I}_{3}$ value at the concentration range higher than $\mathrm{C}_{1}$, indicates that $0.5 \%$ HPC does not change the hydrophobic pocket occupied by the fluorescent probe in any surfactant (Table 1) as observed in the SDS with $0.5 \% \mathrm{PEO}^{[17]}$. However, in the presence of $1.2 \% \mathrm{HPC}$ the $\mu \mathrm{P}$ shifts toward a more polar environment in all surfactants possibly due to increase of the interaction with the polymer segment resulting an aggregate more penetrable to water ${ }^{[18]}$. Otherwise, the $\mu \mathrm{P}$ decreases again about [SDS] 6 and $10 \mathrm{mmol} . \mathrm{L}^{-1}$ in the presence of 0.5 and $1.2 \% \mathrm{HPC}$, respectively. These values are assigned to the saturation concentration $\left(\mathrm{C}_{2}\right)$ related to onset of free micelle formation that depends linearly on polymer concentration $^{[19]}$ as observed. At $298 \mathrm{~K}$, $\mathrm{C}_{2}$ values are difficult to devise for CS or DC/HPC systems (Figure 2).

Increasing the temperature contributes to aggregation disrupting the solvating shell around the molecules of the solute and solvent. In terms of the surfactant monomer and the macromolecule (solutes) there is an effective reduction of the polarity making easier the interaction between the hydrophobic molecular regions ${ }^{[20,21]}$. On the other hand, there is the breakdown of the three-dimensional structure of water and, consequently, weakness of the hydrophobic interactions ${ }^{[22]}$. The former effect is expected to decrease the critical surfactant concentration as observed for SDS in the presence of ethyl(hydroxyethyl) cellulose close to semi-dilute regime ${ }^{[20,21]}$ whereas the latter effect increases the solubility of the solute, particularly the surfactant monomer as observed by the increase of the $\mathrm{cmc}^{[6,13]}$.

Regarding less the small difference between the 
Table 1. Aggregation Parameters from Fluorescence Measurements

\begin{tabular}{|c|c|c|c|c|}
\hline \multirow[t]{2}{*}{$\mathbf{T}, \mathbf{K}$} & \multirow[t]{2}{*}{$\begin{array}{c}\text { System }^{\mathrm{a}} \text { in } \\
\mathrm{NaCl} 0.1 \mathrm{~mol}^{-1} \mathrm{~L}_{-1}\end{array}$} & \multirow[t]{2}{*}{$\mu \mathbf{P}^{\mathbf{b}}$} & \multicolumn{2}{|c|}{$\begin{array}{c}\mathbf{C}^{\mathrm{c}} \\
\text { mmol. } \mathbf{L}^{-1}\end{array}$} \\
\hline & & & $\mathbf{A}^{\mathbf{d}}$ & $\mathbf{B}^{\mathbf{d}}$ \\
\hline \multirow{3}{*}{298} & SDS, & 1.12 & 1.0 & 2.0 \\
\hline & SDS, HPC $0.5 \%$ & 1.12 & 0.63 & 1.0 \\
\hline & SDS, HPC $1.2 \%$ & 1.15 & 1.1 & 2.2 \\
\hline \multirow{3}{*}{312} & SDS, & 1.08 & 1.4 & 2.0 \\
\hline & SDS, HPC $0.5 \%$ & 1.08 & 0.57 & 1.1 \\
\hline & SDS, HPC $1.2 \%$ & 1.14 & 1.2 & 2.7 \\
\hline \multirow{3}{*}{298} & DC & 0.68 & 2.3 & 3.1 \\
\hline & DC, HPC $0.5 \%$ & 0.67 & 0.78 & 1.1 \\
\hline & DC, HPC $1.2 \%$ & 0.75 & 1.4 & 3.2 \\
\hline \multirow{3}{*}{312} & DC & 0.66 & 2.4 & 3.3 \\
\hline & DC, HPC $0.5 \%$ & 0.70 & 1.1 & 2.1 \\
\hline & DC, HPC $1.2 \%$ & 0.88 & 2.3 & 3.7 \\
\hline \multirow{3}{*}{298} & $\mathrm{CS}$ & 0.78 & 6.5 & 9.3 \\
\hline & CS, HPC $0.5 \%$ & 0.75 & 7.0 & 12 \\
\hline & CS, HPC $1.2 \%$ & 0.84 & 8.0 & 13.3 \\
\hline \multirow{3}{*}{312} & $\mathrm{CS}$ & 0.76 & 6.8 & 9.7 \\
\hline & CS, HPC $0.5 \%$ & 0.78 & 7.2 & 14 \\
\hline & CS, HPC $1.2 \%$ & 0.86 & 7.8 & 15.8 \\
\hline
\end{tabular}

a)HPC 153,000, w/v.; b) Micropolarity, $\mu \mathrm{P}$, is the bottom average value of the ratio $\mathrm{I}_{1} / \mathrm{I}_{3}\left(\mathrm{Py}^{1 *}\right)$ in the Figure 1 , error $\left.\pm 0.02 ; \mathrm{c}\right) \mathrm{C}$ is the critical micelle $(\mathrm{cmc})$ or aggregation $\left(\mathrm{C}_{1}\right)$ concentration, in $\mathrm{mmol} . \mathrm{L}^{-1}$, error $\pm 10 \%$; d) obtained as (A) by the inflection point of aggregation curves calculated from the minimum of its first derivative, or (B) by the intercept from linear extrapolation on bottom and steep data.

temperatures employed here (limited by the low cloud point of the sample) the data in the Table 1 indicate a trend toward the increase of $\mathrm{C}_{1}$ with the temperature for all systems, most to CS and DC than SDS. In addition, at $312 \mathrm{~K}$ the $\mathrm{C}_{2}$ values become apparent for all systems specially with $1.2 \%$ HPC for CS, DC and SDS as 30,12 , and 5 mmol. $\mathrm{L}^{-1}$, respectively. The temperature effect on shortening the SDS binding range for $1.2 \% \mathrm{HPC} / \mathrm{SDS}$ system agrees with reported SDS/PEO behavior ${ }^{[23]}$. Moreover, from 298 to $312 \mathrm{~K}$ despite the experimental error, the $\mu \mathrm{P}$ changes differently in the aggregates up to $\mathrm{C}_{2}$ as it decreases slightly for SDS/HPC systems whereas it increases for DC and CS/HPC systems.

A general feature for all aggregation curves (Figures 2) is the stepwise behavior observed. It is related to cooperative process that is more pronounced as sharper is the steeper segment in the curve. Therefore, the HPC seems to reduce the cooperative degree of the aggregation for all surfactants as reported for other SDS/polymers systems ${ }^{[21]}$.

Viscosity: At $298 \mathrm{~K}$ the relative viscosity of $0.5 \%$ HPC as a function of surfactant concentration is presented in Figure 3. At low surfactant concentrations (bellow $\mathrm{C}_{1}$ ) the viscosity is mainly modified for SDS/ HPC system whereas there is a small decrease for the bile salts/HPC systems (Figure 3 ). In addition, the $\eta_{\text {rel }}$ values for SDS/HPC system at surfactant concentrations above $\mathrm{C}_{1}$ differ also from those for DC or CS/HPC. The curve for SDS /HPC system has the same shape that was reported without salt ${ }^{[24]}$ changing the maximum position to lower SDS concentration as expected by the ionic strength effect ${ }^{[25]}$. The maximum viscosity at about 4 mmol.L-1 SDS is the same value observed for SDS/ ethyl(hydroxyethyl) cellulose systems independently of the polymer concentration ${ }^{[4,19,26]}$. The occurrence of the maximum viscosity can be attributed to higher content of polymer segments in the SDS aggregates with a few surfactant monomers as it should occur in concentration range immediately above $C_{1}{ }^{[2,26]}$. The subsequent decrease in the viscosity with increasing SDS concentration indicates that the aggregate formation leads to a more compact polymer structure due to reduction of the level of entanglement or cross linking

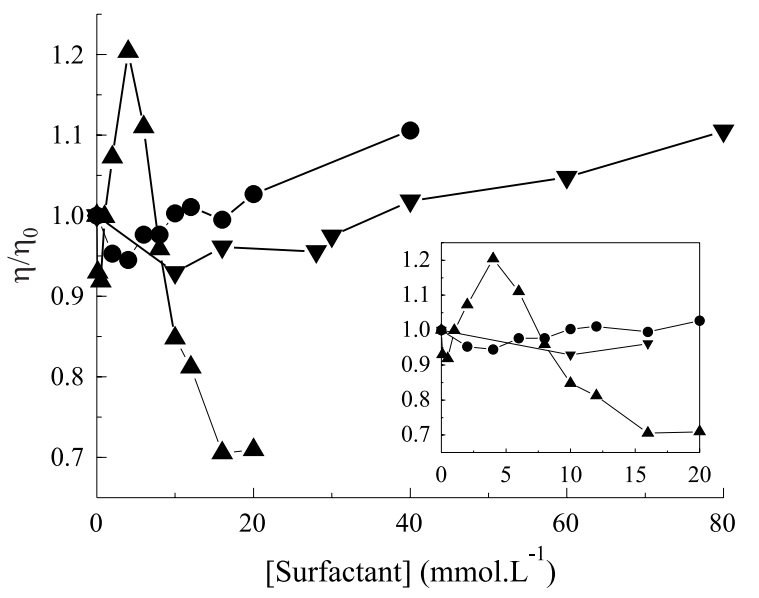

Figure 3. Relative viscosity, $\eta_{\text {rel }}=\eta / \eta_{0}$ of $0.5 \% \mathrm{w} / \mathrm{v} \mathrm{HPC}$ as a function of surfactant concentration SDS $(\mathbf{\Lambda})$, DC $(\bullet)$; and $\mathrm{CS}(\boldsymbol{\nabla})$. NaCl 0.1 mol.L ${ }^{-1}$ at $298 \mathrm{~K}$. Insert: the same data at short range surfactant concentration up to $20 \mathrm{mmol} . \mathrm{L}^{-1}$ 
as the polymer substituent groups redistribute themselves in the increasing number of aggregates ${ }^{[2,26]}$. At higher SDS concentration the viscosity falls below the initial level, without SDS, indicating the increase of the degree of flexibility/mobility as result of the HPC becoming more hydrophilic ${ }^{[2]}$.

On the other hand, the relative viscosity for $0.5 \%$ HPC solutions show an upward jump above $\mathrm{C}_{1}$ for $\mathrm{DC}$ and CS and then a steady increase. It is worth to note that the sharpness on viscosity increase reflects the inverse order of the surfactant monomer aqueous solubility, a fact that can correlate the intensity of the interaction surfactant/HPC. An earlier study ${ }^{[24]}$ with HPC compared SDS with alkyltrimethylamonium halide surfactants indicating a stronger interaction of the SDS than the cationic surfactants. Moreover, some cationic surfactants (dodecyl derivatives) showed a distinct interaction as a slight increase of the viscosity attributed to small size of their aggregates. Indeed, the free micelles of the bile salts or even in the presence of PEO and PVP are smaller than the correspondent SDS systems. ${ }^{[7,8,13]}$

Dynamic Light Scattering: Figures 4 show the angle dependence for relaxation rate of the slow mode, $\Gamma_{2}$, and of the fast one, $\Gamma_{1}$, for different $0.5 \% \mathrm{HPC} / \mathrm{NaCl}$ $0.1 \mathrm{~mol}^{-\mathrm{L}^{-1}}$ solutions with and without surfactants, calculated from a two-exponential fit. Each system presents two components as assigned to polymer chain or polymer/surfactant aggregate (fast) and some polymer aggregate (slow) that is minimal due to dilute regime adopted. Both modes are diffusive as the plots of the relaxation rate, $\Gamma$ versus the square of scattering vector, $\mathrm{q}^{2}$, pass through the origin for all systems studied. Considering the slow mode, the SDS addition shows the highest diffusion coefficient among the systems presented. On the other hand, the fast mode of the polymer chain is also turned faster by both surfactants but mainly by the addition of DC. Regarding that both surfactant concentrations are immediately above $\mathrm{C}_{1}$, these results indicate the effectiveness of the big SDS aggregates to redistribute the HPC polymer segments eventually entangled whereas the small DC aggregates can just affect the flexibility/mobility of the single HPC chain. Indeed, the relative viscosity measurements agree with that behavior at each surfactant concentration.

To summarize the main features in this study we recall the possible forms of interaction between a nonionic polymer and an ionic surfactant: (a) redistribution of the surfactant between the bulk solution and the coil regions; (b) surfactant molecules bound
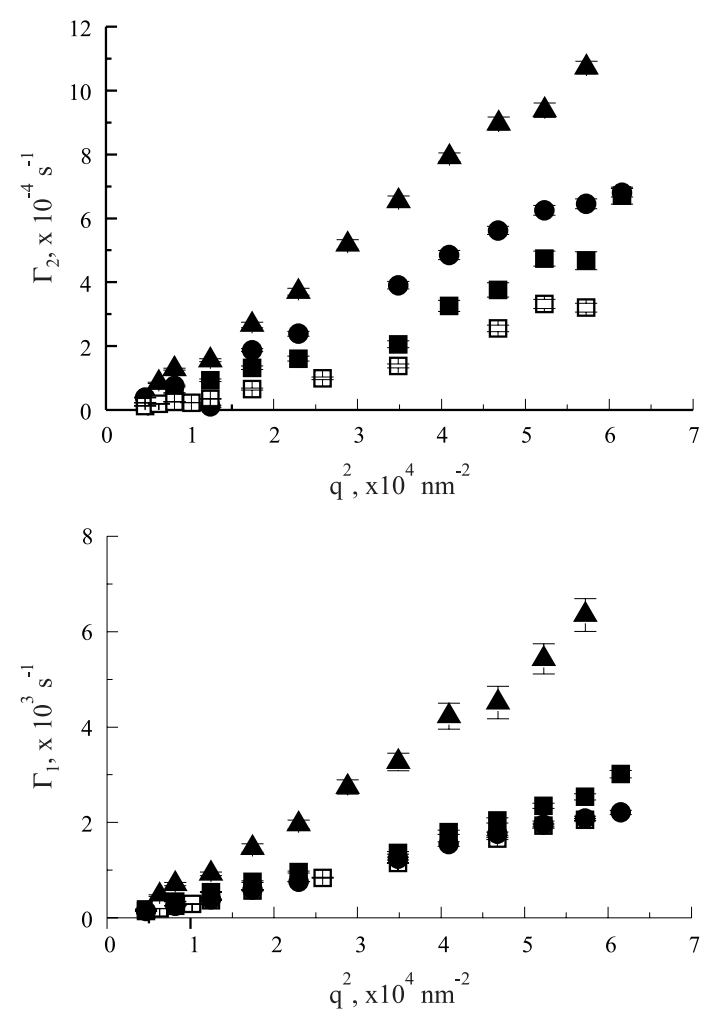

Figure 4. Angle dependence of the relaxation rate, $\Gamma$, for $0.5 \mathrm{~g} . \mathrm{dL}^{-1}$ HPC ( $\square$ ) plus SDS $5 \mathrm{mmol} . \mathrm{L}^{-1}(\bullet)$, DC $5 \mathrm{mmol.L} \mathrm{L}^{-1}(\bullet)$, and CS 17 mmol.L-1 $(\mathbf{\Lambda})$. On top, $\Gamma_{2}$ slow mode, on bottom, $\Gamma_{1}$ fast mode.

individually along the polymer chain; (c) surfactant molecules clustered around hydrophobic sites on the polymer; (d) polymer molecules wrapped around surfactant micelles in such a way that the polymer segments partially penetrate and wrap around the polar head group regions of the micelles ${ }^{[19]}$. The present data set points out that a combined and adjustable formation could better explain the aggregation behavior these surfactant/HPC systems that correlates the aqueous solubility of the surfactants: SDS $<\mathrm{DC}<\mathrm{CS}$. In the SDS/ HPC system, the interaction means that the addition of the more hydrophobic, flexible and charged surfactant, SDS is redistributed to HPC in the form of aggregates on the polymer backbone with continuously solubilization of the polymer ${ }^{[15,27,24]}$. The increase micropolarity and relative viscosity of the free polymer solution reflects it as well as the decrease of the cmc related to SDS free micelle indicates it. Differently, the interaction of the CS with HPC can be described as it is initially redistributed as surfactant monomer along the polymer chain followed by the aggregate formation and partial polymer solubilization. At concentration immediately above $\mathrm{C}_{1}$, the decrease of the viscosity for $\mathrm{CS} / 0.5 \% \mathrm{HPC}$ solutions indicates that a more compact 
polymer chain formation. The small effects on increasing the $\mathrm{cmc}$ and decreasing the microviscosity related to CS micelle reflect a weak interaction of the aggregates and the HPC chain. Indeed, the partial polymer solubilization could be understood as its adsorption on the hydrophobic exposed faces of the CS aggregates that is about $60-80 \%$ of total hydrophobic surface ${ }^{[6]}$. The DC aggregation behavior is intermediary between SDS and CS but closer to the latter as expected by molecular resemblance. The picture is complete as the hydrophobic exposed surface increases for all systems as the temperature and polymer concentration increases. The latter conditions were observed by fluorescence measurements indicating that the parameters $\mathrm{C}_{1}, \mu \mathrm{P}$ and $\mathrm{C}_{2}$ are shifted toward the redistribution of the aggregates along the polymer chain: delaying the aggregate formation $\left(>\mathrm{C}_{1}\right)$, loosing the aggregates $(<\mu \mathrm{P})$ and shortening the polymer saturation length $\left(<\mathrm{C}_{2}\right)$. It is possible that in the later conditions the aggregation mechanism is changing from associative to segregative type ${ }^{[28]}$. It is worth to note that the moderate ionic strength employed avoid that the strong electrostatic interactions override the role of the hydrophobic and weak London dispersive forces on the aggregation behavior. Finally, the study points out a relation between the aggregation mechanism to anionic surfactant/non ionic polymer aqueous systems and the solubility, shape and geometry of the surfactants. The extension of the temperature and polymer concentration effects to other techniques is currently investigated as well as the aggregation number parameter.

\section{Conclusions}

The anionic surfactants SDS, CS and DC have shown different aggregate behavior in the presence of HPC. At this point, the interaction of SDS with HPC is being more effective than CS or DC. Besides the charge, size and geometry of the surfactants, and the rigidity of HPC may play a role in order to expose the hydrophobic segments to weak interaction with the bile salts, CS and DC.

\section{Acknowledgements}

Capes, CNPq, Fapergs, Propesq-UFRGS.

\section{References}

1. Winnik, F. M.; Regismond, S. T. A. - Colloids Surf.A, 118, 1-39 (1996).
2. Singh, S. K.; Nilsson, S. - J. Colloid Interface Sci., 213, 152-159, (1999).

3. Anthony, O.; Zana, R. - Langmuir, 10, 4048-4052, (1994).

4. Evertsson, H.; Nilsson, S. Carbohydr. Polym. 1998, $35,135-144$.

5. Aoudia, M.; Zana, R. - J. Colloid Interface Sci., 206, 158-167, (1998).

6. Garidel, P.; Hildebrand, A.; Neubert, R.; Blume, A. Langmuir 2000, 16, 5267-5275.

7. Christoff, M.; daSilveira, N. P.; Samios, D. - Langmuir, 17, 2885-2888, (2001).

8. Christoff, M.; Mezzalira, G.; daSilveira, N. P.; Samios, D. J. - Colloid Interface Sci., submitted, (2002).

9. Small, D. M. - In The Bile Salts; Nair, P. P., D.Kritchevsky, Eds.; Plenum Press: New York, pp 249-256, (1971).

10. Yim, C. T. - Langmuir, 13, 4383-4390, (1997).

11. Kalyanasundaram, K.; Thomas, J. K. - J. Am. Chem. Soc. 1977, 99, 2039-2044.

12. Provencher, S. W. - Comput. Phys. Commun., 27, 229242, (1982).

13. Paula, S.; Sus, W.; Tuchtenhagen, J.; Blume, A. - J. Phys. Chem., 99, 11742-11751, (1995).

14. Persson, B.; Nilsson, S.; Sundelof, L.-O. - Carbohydr. Polym., 29, 119-127, (1996).

15. Winnik, F. M.; Winnik, M. A.; Tazuke, S. - J. Phys. Chem., 91, 594-597, (1987).

16. Brackman, J. C.; Engberts, F. N. - Chem. Soc. Rev., 22, 85-92, (1993).

17. Zana, R.; Lianos, P.; Lang, J. - J. Phys. Chem, 89, 4144, (1985).

18. Stam, J. V.; Brown, W.; Fundin, J.; Almgren, M.; Lindblad, C. - In Colloid-Polymer Interactions; Dubin, P. L., Tong, P., Eds.; American Chemical Society: Washington, DC, Vol. 532; pp 195-215, (1993).

19. Holmberg, C.; Nilsson, S.; Singh, S. K.; Sundelöf, L.O. - J. Phys. Chem., 96, 871-876, (1992).

20. Kamenka, N.; Burgaud, I.; Zana, R.; Lindman, B. - J. Phys. Chem., 98, 6785-6789 (1994).

21. Singh, S. K.; Nilsson, S. - J. Colloid Interface Sci., 213, 133-151, (1999).

22. Muller, N. - Acc. Chem. Res., 23, 23-28, (1990).

23. Takasawa, Y.; Ueno, M.; Meguro, K. - J. Colloid Interface Sci., 78, 207-211, (1980).

24. Drummond, C. J.; Albers, S.; Furlong, D. N. - Colloids Surf. A, 62, 75-95, (1992).

25. Evertsson, H.; Holmberg, C. - Colloid Polym. Sci., 275, 830-840, (1997).

26. Wang, G.; Olofsson, G. - J. Phys. Chem., 99, 55885596 (1995).

27. Suto, S.; Iwasawa, I. - J. Polym. Sci. A, 31, 1599-1607, (1993).

28. Piculell, L.; Bergfeldt, K.; Gerdes, S. - J. Phys. Chem., 100, 3675-3679, (1996).

Recebido: $15 / 08 / 01$

Aprovado: 06/04/02 